\title{
CEREBRAL VENOUS THROMBOSIS: AN EXPERIENCE OF A TUNISIAN PEDIATRIC DEPARTEMENT
}

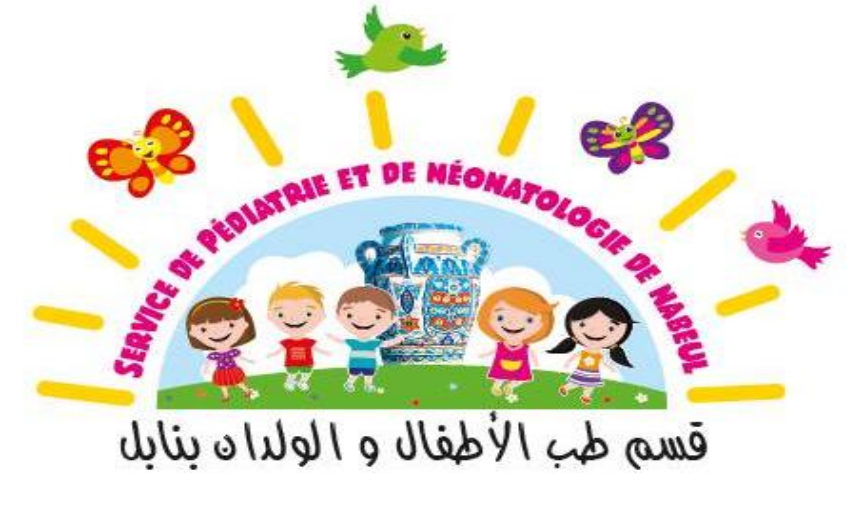

Guedria $\mathrm{A}^{1}$, Abdelkarim R${ }^{1}$, BachroucheH ${ }^{1}$, Chraiet Rezgani ${ }^{1}{ }^{1}$, Bouzaidi $\mathrm{Kh}^{2}$, Aissa $\mathrm{Kh}^{1}$

Tunis El Manar University, Faculty of Medicine of Tunis

1: Pediatric department, Mohamed Tlatli hospital, Nabeul

2: Medical imaging department, Mohamed Taher Maamouri hospital, Nabeul, Tunisia

\section{I/Background:}

$>$ Cerebral venous thrombosis (CVT) is a rare and potentially life-threatening condition in the pediatric population that may have vital or morbid consequences.

$>$ The available data suggest an incidence of 0.67 cases per 100,000 children per year.

D The prevalence of childhood CVT varies according to age; neonates are the most commonly affected.

$>$ The clinical presentation is frequently non specific, thus the diagnosis is often delayed or missed.

\section{II/Methods:}

$>$ Retrospective chart review: the medical records of all children admitted in our center with CVT during the period August 2016- April 2018 were reviewed.

$>$ Data concerning clinical presentation, treatment and outcomes were reviewed and analyzed.

\section{III/Results:}

$>$ Six patients were identified : 3 infants (range 2-6 months) and 3 childs (range 9-11 years).

$>$ Presenting symptoms were non specific: The most common was fever.

$>$ Demographics and clinical data Table I

\begin{tabular}{|c|c|c|c|}
\hline Case $n^{\circ}$ & Age at onset & Sex & Symptoms \\
\hline 1 & 51 days & M & $\begin{array}{c}\text { Fever, lethargy, Hypernatremic dehydration, } \\
\text { nystagmus,Boxing and bicycling }\end{array}$ \\
\hline 2 & 60 days & M & Fever, apathy, peripheral facial paralysis \\
\hline 3 & 6 months & $\mathrm{F}$ & Fever, Bottle Refusal, seizure \\
\hline 4 & 9 years & $\mathrm{F}$ & $\begin{array}{c}\text { Fever, Otalgia,Vomiting, thrombocytopenic } \\
\text { purpura }\end{array}$ \\
\hline 5 & 11 years & M & Fever, Headache, Vomiting, Palpebral edema \\
\hline 6 & 11 years & M & Fever, Otorrhea, Torticollis \\
\hline
\end{tabular}

Table I:Demographics and clinical data

$>$ An urgent contrast-enhanced CT of the head and neck was immediately performed for all patients, revealing cerebral venous thrombosis which has been confirmed by MRI

$>$ Territory of CVT

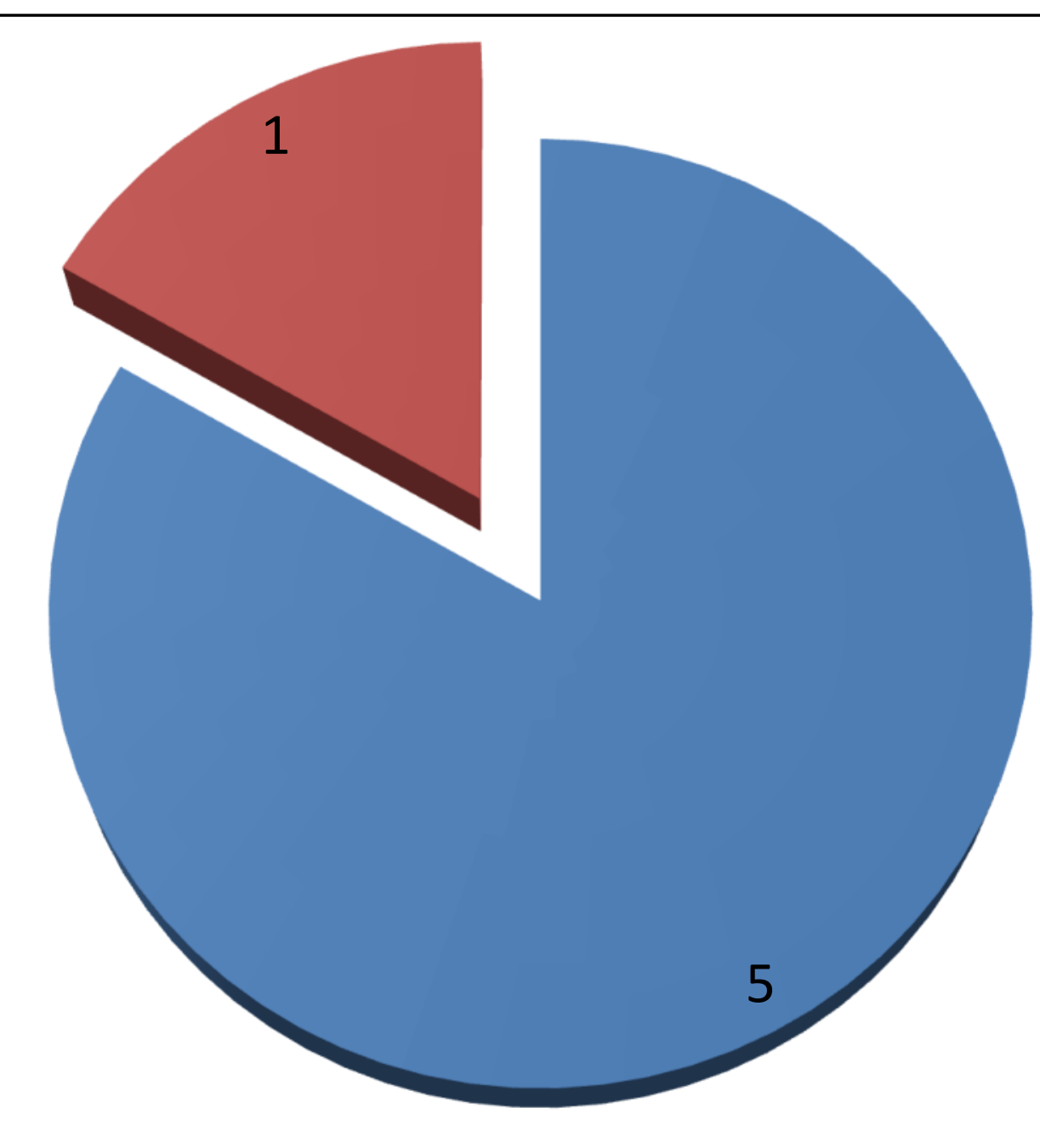

- INTERNAL JUGULAR VEIN

Figure 1: Territory of the CVT

\section{$>$ Causes of CVT}

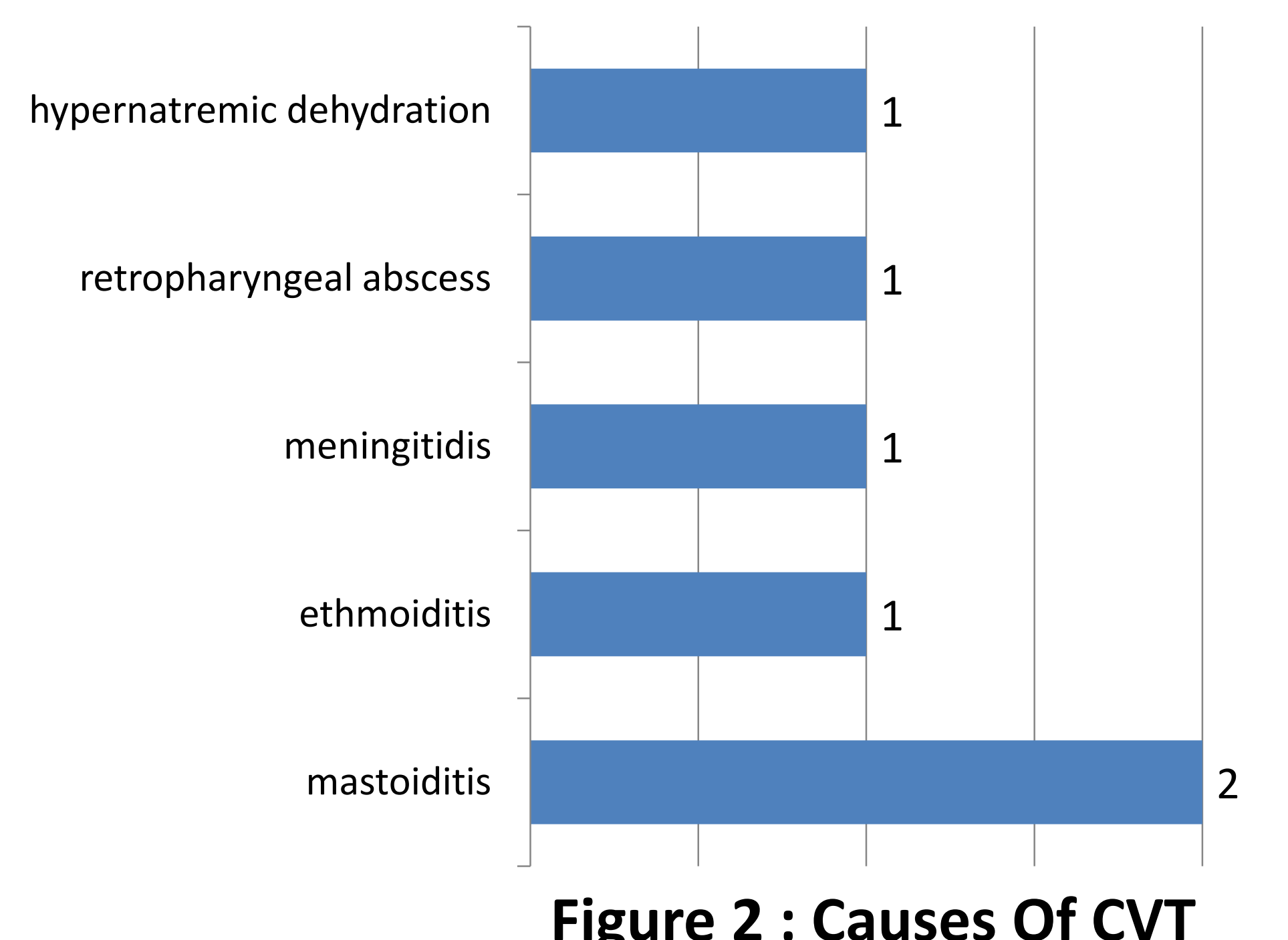

$>$ All patients were treated with appropriate antibiotics.

$>$ Five patients were treated with anticoagulation (low molecular weight heparin) with a relay by the Vitamin $\mathrm{K}$ antagonists (VKA).
> None of the patients treated with anticoagulation therapy had clinically significant bleeding complications.

$>$ Evaluation of thrombophilia was performed in all patients.

$>$ One patient (case 2) had protein $\mathrm{C}$ deficiency

$>$ Developmental outcome at the most recent examination revealed normal development in 3 of 6 patients,growth delay and poor school performance in 1 patient.

$>2$ infants died (case 1 and 2).

\section{IV /Conclusions :}

> CVT may be caused by hypercoagulable states, by conditions causing blood flow disturbances, or by inflammation/infection.

$>$ In our study, we observed more than one risk factor that predisposed to CVT.

We speculate that the genetic prothrombotic condition predisposed to CVT, which was triggered by a central nervous system infection and otogenic infections.

$>$ Thus, thrombophilic evaluation should be encouraged in all children with CVT, in order to identify those with increased risk factors for thrombophilia.

> The percentage of patients with CVT who have an identified risk factor for thrombophilia varies significantly from a study to another, it ranges between $33 \%$ to $99 \%$. A German study reported cases in which either an elevated lipoprotein A or a protein C deficiency was associated with CVT; in an American study, protein $\mathrm{C}$ and antithrombin III deficiencies were common .

$>$ CVT may be delayed and even missed. Clinicians must maintain a high index of suspicion when treating any child with an acute central nervous system infection or otogenic infections and presenting neurological signs and symptoms despite administration of appropriate therapy.

$>$ The currently available diagnostic tools include head CT and MRI.

$>$ The mains stay of CVT treatment is anticoagulation. The purpose of anticoagulant therapy is to limit the propagation of an existing clot and to prevent the formation of further thrombi.

$>$ Previous studies have reported encouraging data on the safety of anticoagulation therapy in children with CVT and on improved cognitive outcome.

$>$ Although definitive evidence of effectiveness is lacking, there is a general consensus that anticoagulation with unfractionated intravenous heparin or subcutaneous LMWH is appropriate treatment for acute CVT [14]. The treatment most used for infants and children is LMWH for 7-10 days (120-180 Ul/kg twice a day in children up to 1 year of age, and $100 \mathrm{UI} / \mathrm{Kg}$ twice a day in older patients) followed by oral anticoagulants generally for 3-6 months.

$>$ Finally, CVT in children sometimes has a poor prognosis despite prompt diagnosis and therapy. Commonly identified morbidities include motor dysfunction, neuropsychiatric

disorders, visual impairment, seizures, headaches and even death.

$>$ An early diagnosis led to prompt therapy and consequently enabled a good neurodevelopmental outcome.

\section{V/References:}

R. BenAbdallahChabchouba, ${ }^{*}$, A.Riqueta, A.Ramdaneb, L.Valle'ec, S. Raccoussota: Lemierre syndrome revealed by torticollis

$>$ Bozzola et al; BMC Pediatrics 2014, Multiple cerebral sinus thromboses complicating meningococcal meningitis: a pediatric case report

$>$ Daniel Tibussek MD et al; Late-Onset Group B Streptococcal Meningitis Has

Cerebrovascular Complications ; the Journal of pediatrics

$>$ Brandon Isaacson, MD, Christine Mirabal, MD, J. Walter Kutz, et al, Dallas, TX

Pediatric otogenic intracranial abscesses; ORIGINAL RESEARCH-PEDIATRIC OTOLARYNGOLOGY

Author Names and Contact Details:

email: asmaguedria9@gmail.com 